Information Management and Business Review

Vol. 5, No. 11, pp. 553-560, Nov 2013 (ISSN 2220-3796)

\title{
A Study on the Element of Sentiment toward Knowledge Sharing among Knowledge Workers in a Virtual CoP
}

\author{
Ng Chuee Leng, *Siew Hoong Angela Lee, Tong-Ming Lim \\ Sunway University, Malaysia \\ *angelal@sunway.edu.my
}

\begin{abstract}
Ever since the usage of alternative media has grown tremendously, it was seen that the increasing amount of interactive online sites that are categorized as Web 2.0 systems has changed the communication and collaboration among knowledge workers. These systems provide greater interactivity and higher user-generated content. Social networking site is one type of online platforms that allow people to collaborate and communicate through a variety of services for social purposes (Broughton, Higgins, Hicks, \& Cox, 2009). These high-flying social networking sites are driving new forms of social interaction, allowing users to intermingle and cooperate with each other in a social media dialogue nowadays by swapping ideas or posting updates and comments. It is crucial to comprehend how social relationships affect the content share between each knowledge worker in a virtual world. This project investigates how work and friend relationships can influence the utilization of social media systems for knowledge sharing. The effect of the element of sentiment among knowledge workers in a virtual Community of Practice ( $\mathrm{CoP}$ ) as they send and receive messages with their peers will be studied in this research. Different forms of emotion from knowledge workers will be investigated in this study in the workplace. A proposed research model will be proposed. A research framework will be outlined and qualitative and quantitative analysis will be used to analyze the research results.
\end{abstract}

Keywords: Community of Practice, Sentiment, Knowledge Sharing, Knowledge Workers, Social network sites

\section{Introduction}

It is the desire of many organizations to develop a more effective knowledge sharing culture where knowledge workers collaborate to share their knowledge effectively. Organizational knowledge sharing is the fundamental and vital process for organizations to be more successful in current business eco-system. Knowledge Management (KM) is the formal gathering process that organizes, distributes, and effectively usage of knowledge in the forms of resources and people skills. This helps to develop the competence of the people resources in the organizations by improving the sharing of what they know and making the knowledge easily obtainable or accessible by those who need it. Peariasamy \& Mansor (2008) pointed out that one of the objectives of knowledge management projects is to encourage the sharing of knowledge among employees. More than ever, the success or competitive advantage of many organizations is increasingly hinges on their ability to create and share knowledge efficiently. Their success depends on effective knowledge management and organizational learning. Knowledge sharing is about synergistic alliance of individuals who bears a common goal or ambition by reciprocally exchanging knowledge and creating new knowledge in cooperation (Gagne, 2009). Transitive memory, people, and content are the three elements in knowledge sharing: knowing who knows what and where the information is, with who does the sharing take place and what sort of information is being shared (Nandkeolyar, 2008). The behavior of employees determine the successfulness of knowledge management systems that being practice in a company, hence one should be enthusiastic to share knowledge and treat is as a valuable asset since it is important to achieve a set of objective both personally as well as benefits his organization.

Over the past ten years, the enormous growth of social media related activities and social/professional networks has created new opportunities as well as new challenges for organizations that want to embrace the dynamic world of social interaction and fluid knowledge flows. For example, social networking media tools such as Twitter and Facebook were utilized by employees to promote networking in workplaces. These social tools augment information exchange and knowledge sharing among employees in small-to-large organizations facilitating building of common ground and maintaining a feeling of connectedness among individuals across the organizational hierarchy. Besides, social media can also facilitate the development of employees in terms of connections maintenance with a large group of 
colleagues in a more efficient way (Huy \& Shipilov, 2012). Another benefit of using social media in organizations is that social network allows employees to feel that they are being recognized and appreciated by their own organizations while involving in information sharing or other online activities. This can rectify the shortcomings or deficiency of a company culture indirectly via information exchanged on social networks. Another benefit of social networking tools is their ability to construct bonding between employees, allowing them to put forth to unfamiliar colleagues and to discover experts in the company.

Social network analysis is a classic pattern of thought which can be found and used in numerous fields as it derives from the concept of paths or chains that individuals communicate to exchange information. Otte \& Rousseau (2002) stated that many organizations use informal networks as the primary means by which employees find information, solve complex problems, and learn how to do their work. In Abrams et al. (2003) work, they also pointed out that to communicate in a social network effectively, two forms of interpersonal trust are required - trust in a person's competence and in a person's benevolence - to enable effective knowledge creation and sharing in these networks. This paper aims at investigating the sentiment element among knowledge workers of companies in the process of collaborating and communicating to share knowledge via social network sites. Sentiment analysis offers knowledge workers the ability to keep an eye on sentiment attributes of various comments of participating knowledge workers on the social media sites before taking these fragmented intelligences as reference to judge another participating knowledge worker on their knowledge sharing activity. In the analysis of their knowledge sharing activity, text analytics and sentiment analysis will be used.

\section{Literature Review}

Sentiment Analysis of Ego Network: Social network services allow an individual to connect with another individual of similar interest to share information and opinions. There are various types of social networks, for example the educational network, professional network, and ego social network. Educational networks are educationally-focused networks which functions as the place where students works together on file sharing, discussions or interacts with lecturers via chats or forums mainly on their academic-related work or projects (Hargadon, 2012). A professional network is designed for professional; intend to focus exclusively on interactions and relationships of a business nature. The purpose of professional network is to provide professionals opportunities for career-related growth or look for employment, as well as general forum to share knowledge by connecting on common interests and job posts (Connor, 2012). Arnaboldi et al. (2012) stated that ego networks are social networks that formed by an individual's personality in conjunction with social ties or relation he encompasses with other people, and is particularly shaped out due to similar human cognitive processes between both parties. Furthermore, individuals use online social networks to share own generated content in social sites, indirectly forming ties that bind different individuals up with information exchanged or shared online due to similar thinking ways (Arnaboldi, Conti, Passarella, \& Pezzoni, 2012).

Knowledge sharing on a social network in particular ego centric or professional centric networks (Fisher, 2005) does have rooms for potential organizational development and improvement as it offers chances for employees to understand and improve their performance in their organization through effective communication and knowledge sharing activities. It is hope that the contributions from this study will provide deeper understanding on how the element of sentiment among employees in a social network is able to affect the knowledge sharing and communication hence influence the growth of the companies. Sentiment analysis intends to determine the thoughts or feelings of a speaker or a writer with reverence to certain subject/topic or the overall related division of an object. Tan et al. (2011) defines thoughts as a person's opinion or assessment, emotional condition, or the emotional consequence which author hopes to have on readers. Other than that, sentiment analysis works as one of the vital rising technologies by lending a hand to people to steer the enormous amount of user generated content which obtainable online. Ranging from product evaluations to political news, there are tools that automatically find out the user's perspectives which would allow them to make sense of the massive body of views that have been uttered or expressed on the Internet. Findings regarding social relationships on a social network can be applied in many ways to perk up user-level sentiment analysis. Emotions of Twitter users can be categorized via tweeted posts as users that have related or similar judgments are "connected" in some ways through the use of “@” on network formed by Twitter via textual features (Tan et al., 2011). There are two causes that drive Tan et al. (2011) to suggest a way to make use the information about user-user connection on online social networks to get better sentiment analysis. Foremost, the user-association 
information is easily accessible since user-created content regularly visible in the framework of social media.

An important point to highlight is that, Twitter manages not only tweets but also the list of followers as well as common friends between followers. Furthermore, the links in a network formed by Twitter do create connections between users, and it builds the bonding for a personal relationship as per described by the theory of homophily. The theory of homophily states that individual always associate and link with others of the same kind, in terms of demographic and social features which consist of gender, race, geographic location, and group assignment (Yuan \& Gay, 2006). Homophily implies that users that are "connected" by a mutual personal relationship are more likely to hold parallel opinions. Homophily works on both positive and negative response, as the connection which users form may keep up a correspondence to endorsement. For instance, a well-known figure or a phrase that is attention paying aspiration might arouse echo within users which shares the same interest or same ideas across social network sites. Generally, once an individual distinguish if there are some traits that others have in common with themselves, they tend to have positive judgement and do pay attention to that particular attribute. The occurrence of homophily and relationship tie among individuals within the equal network is due to the attraction of similarity standpoint (De Choudhury, 2011). So as the resonance exists among workers within same company, it is much easier for them to share knowledge and communicates in virtual Community of Practice (CoP).

Knowledge Sharing Related Works: Every successful enterprise or organization constantly enhances work efficiency by exchanging knowledge. The process that people obtain knowledge and being educated on others' experience can be exchanged via the intranet, extranet or Internet. It is very important for employees to share knowledge efficiently or unselfishly within an organization, since knowledge is the power for a company. In addition, companies will gain more benefits and improvements especially when their employees are willing to share or exchange their knowledge with other colleagues. The basis of organizational knowledge creation is the conversion of tacit knowledge into explicit knowledge and back again. Explicit knowledge is knowledge that "can be expressed in words and numbers, and easily communicated and shared in the form of hard data, scientific formulae, codified procedures, or universal principles". Examples of explicit knowledge include chemical formulae, market forecasts, operations procedures, product specifications, software code, and technical standards. Do not view tacit knowledge and explicit knowledge as mutually exclusive but as complementary entities. Over time, human knowledge shifts between the tacit and the explicit through a process of social interaction between individuals that also produces new knowledge and expands its use. However, there are some barriers that influence employees on knowledge sharing although it is being recognized as an essential asset in companies nowadays. An example is that employees that work in traditional companies treat their learned knowledge as the source of power and will not share their knowledge with other colleagues easily since they are afraid that it will influence their current status in the company. Another aspect is the lack of trust and also different organizational culture background of each employee does affect the efficiency of knowledge sharing process. Employees that come from different organizational culture background often causes misunderstandings during discussions begin; and employees that do not trust outsiders will not have the willingness to share knowledge easily as well.

According to the research conducted by IBM Institute for Knowledge-Based Organizations (2002), they found that there are four diverse relationship elements which play a vital role for effective learning. It is hard to have a person that carries adequate knowledge to resolve ever-increasing and complex problems, especially when we are moving into a knowledge-demanding economy nowadays. The growth of social network discipline has been supported by three vital developments in the management of business world. Firstly, the discovery of informal structure that coexists together with the formal structure within an organization is very important. An example is that, within a same big company individual does have frequent interactions or communications with other department of peoples which is not stated initially within an organization chart. Secondly, the shift of a flatter organizational model which is more flexible, team-oriented and more dependent on knowledge assets requires the understanding of how these structures work and how to manage them. Thirdly, the express growth in close cooperative relationships across organizational boundaries do affects the growth of social network discipline as well. Factors like culture differences, sharing reluctance, lacking of interactions, time and trust serve as barriers that hinder knowledge sharing process among workers within an organization. It is a common phenomenon that knowledge sources voluntarily share knowledge if it is work-related-necessity or only with those that they wish to encourage, mostly colleagues who have good relationship with them. The multi-dimensional 
feeling of trust is crucial to decide on how keen and how much worker shares accurate information, thus making effective communication as one of the basic attribute for efficient knowledge sharing.

Collaboration and Communication in Knowledge Exchange among Knowledge Workers: The understanding on the properties of collaboration and communication among knowledge workers as ties in a social network is of high importance. Using social network as a platform to exchange knowledge among knowledge workers in a virtual Community of Practice (CoP) is also a challenge where actors as ties that are usually not known to each other due to distance and lack of face-to-face interaction. The following examine characteristics, factors, challenges and unsolved issues carried out by researchers. Social network research presupposes that trust is linked by positive ties between two network actors. Chua, Ingram \& Morris (2005) tried to bring out the amount of trust ego that has in alter, in a more detail way by studying on manager's ego-centric professional networks: trust can be built on either a socioemotional basis (affect-based trust) or an instrumental basis (cognition-based trust). These two types of trust lead to diverse results in both laboratory or field settings, as they influenced in or hold back knowledge creation and transfer among workers via Social Network Analysis (IBM Institute for Knowledge-Based Organizations, 2002). This is attributable to different manners by various factors like kind of exchange relationships that exist between them.

Individuals' attitude influences the way how they share knowledge regardless in individual or organizational characteristics. In the study carried out by Hooff and Hendrix (2004) on the differences between the willingness to share and the eagerness to share. Both eagerness and willingness to share are positively related to knowledge sharing - both donating and collecting knowledge. While on the other hand, the difference between willingness and eagerness is that: willingness implies to the readiness of one's mind to get something done while eagerness indicates that one's showing keen and enthusiastic desire or passion to do something. Eventually, their observed results illustrate a division in attitudes which is alike with the eagerness/ willingness: willing individuals most likely to collect more knowledge than they contribute, while eager individuals contribute and collect an equivalent amounts. At the same time, eagerness is completely related to knowledge contributing, whereas willingness is related to both contributing and collecting (Hooff \& Hendrix, 2004). Wasko and Faraj (2005) states that individual motivations like reputation and self-efficacy is one of the reason that causes people to have the willingness to share knowledge with others. Individual who is willing to interact or work together with others is able to obtain more advantages in many aspects, according to Social Exchange Theory (Sun et al., 2010). For instance, people can expand on their own recognition, social status and also reputation by sharing their knowledge. Reputation plays a vital asset for everyone since it picks up or sustains our social status and people who participates in a community action regularly gains more reputation too. The perception contribution and status in the profession may inspire individuals to contribute their precious, personal knowledge to others in the network if an individual wish to gain more reputation. Other than that, individuals can contribute their knowledge in the communities as they think that helping others that with challenging problems or those that are in need is interesting. Thus, individuals will feel satisfied with serving other people if they do not anticipate for any repayment.

Nevertheless, there are quite a few numbers of factors that hold back the willingness of knowledge sharing. An obvious example is that experts with exceptional or extra knowledge who have the highest status plays the role of monopoly control on certain knowledge, creates hoarding instead of sharing. In a more explicit way, knowledge hoarding is the actions which people decides to bound or forbid other social participants from attaining such solely held assets of knowledge (Sun et al., 2010). One of the reasons will be caused by the sensitiveness of data sharing within workplaces or some personal issue within the expert him or herself. Consequently, these knowledge workers would be extra careful to contribute or share knowledge with their colleagues as they are afraid of losing a chance of individual lead and they believe that sharing of one's personal knowledge possibly will cause a relative loss of power within an organization. Statements mentioned shows that knowledge sharing probably put the power or area of expertise of an individual at risk (Sun et al., 2010). The probability of knowledge gaps increasing will occur as if knowledge sharing is restricted across an organization, and these gaps will then turn out to have less-than-desirable working results. It is a natural human affinity to hoard knowledge or when ones shared knowledge to an individual. An additional factor is that: emotions affects knowledge sharing of knowledge workers within an organization too. Emotions influences and manipulates according to human's thought and behaviour. Formerly, emotions or sentiments of worker is not noticeable as the factor which will affect ones performance in the workplace but nowadays interpersonal interactions like employees' emotion directly influences key organizational outcomes (Ghalandari et al., 2012). The 
expression of emotions has also been associated the mood of each employee, as well as the willingness to share or exchange information online via social medias. On top of that, it might be both intentionally or non-intentionally in sharing knowledge as it affects by one's sentiments, thoughts, and accomplishments as well.

Through the literature found on organizational behaviour by De Choudhury and Counts (2013), there are three analysis of emotional expression among employees: its relationship to exogenous/lifestyle and endogenous workplace factors, geography, and organizational structure (De Choudhury \& Counts, 2013). Employees inclined to make significant adjustments in affect expression at the time they work together with others over the organizational hierarchy, and they even pose optimistic affect all the way through interpersonal communications with colleagues in different geographic regions. Their findings have proved that emotions of employees do affects on the productivity of their works; hence positive attitude is encouraged to be encompassed in an organization. There are quite a number of organizations starting to keep up the use of public with tools that allows public to leave comments on, to keep an eye on the conversation channels and distinguish speakers' level of involvement (De Choudhury \& Counts, 2013). In reality, usage of social media in enterprises does impact on the information exchanging, association, communication, and interpersonal connectivity in certain bureau. Nevertheless, these come into a disappointment as it brings uncertain on the effectiveness in capturing organization-precisely sentiment of employees since these tools are basically opens for public. What's more, some of the employees might make use of these social media tools for both private and professional updates or probably do not like to talk about company-related information or jobs in an open forum.

Other than online social media, it comes into a deliberation for De Choudhury and Counts (2013) to conduct a research on how organizations affect their workers and also in reversed way, with respect to workers' thoughts and emotions. The cycles of sentiments among workers do relate directly to their daily life, biological, and sociocultural on top of the inborn personal and behavioural characteristics which act as vital role in stress and job related concerns. These serve as the exogenous and endogenous workplace factors which had been studied. Another consideration is that the impact of collaborative norms in terms with the geographic distancing and work environments are frequently noticeable through expressions of workers affect, as lots of big companies do have offices globally or being outsourced to different countries for work. As a result of this, so as to perk up the communication exchange and encourage innovative idea sharing, it is essential for organizations to be aware of the affective expressions of their workers in and between geographic locations. Furthermore, the process of leading and leader outcomes genuinely interlinked with workers' emotions as well. For instance a leader in negative temper does spread the negative energy or unhappy mood to their employees, as they show signs of mood convergence with respect to workers, workplace norms, tasks, and social interdependence. In a nutshell, the closeness or intimacy of working relationship ties and friendship ties were determined by the level of trust among each worker within an organization. The higher the interaction frequency, the more frequent workers communicate to share information and more willing to exchange various types of knowledge.

\section{Methodology}

The research data for this study will be gathered through interview that carries out after questionnaire survey on the research subject "Influences of Sentiment in Knowledge Sharing". Survey questionnaire will then send to IBM Malaysia internal forums to solicit responses from a focus group consisting of 250 employees from IBM Malaysia Sdn. Bhd. It will be chosen for the reason that they have a packaged social media analytics solution named Cognos Consumer Insight (CCI) that facilitates organizations on understanding customer sentiment. It automatically collect customer generated content from the Internet; evaluate customer sentiment, and identifies customers' preferences and behaviour. Last but not least, text analytics and sentiment analysis will be used to analyze text and sentiments in the text that being posted from the Internet as well. Text analysis fosters qualitative research as it analyze on massive amounts of respondents' textual replies to obtain insight into their sentiment, attitudes, behaviours, thoughts, incentives and background. (Rouse, 2012) Furthermore, the hypotheses of this research will be analyzed by using Statistical Analysis System (SAS) software with Structural Equation Modelling (SEM) as the analysis tool to study the influence of sentiments to knowledge sharing between knowledge workers.

Interview: Pre-data and post-data collection interview will be conducted within 5\% random participants to obtain a more accurate analysis for this research study. The purpose of pre-data collation interview is to seek preliminary understanding of the interviewee's working activities as well as their satisfaction 
towards their current job. As a result, interviewed content serves as the base requirements or information to be consider for questionnaire design later on. Post-data collection interview will be carry out right after questionnaire survey has been done to clarify doubts and provide detail understanding of the responses collected. Participants will then be kept anonymous throughout the whole interview process to ensure information provided will not put their career in the organization at risk. On top of that, recorded interview contents will be transcribed and notes will be taken for this interview to make sure its accurateness.

Questionnaire: The proposed hypotheses devised in this research study will be tested during this stage of the analysis. The survey questionnaire uses the results of pre-data collection interview as the basis in addition to the related works review in the research to design on the instrument, and the target focus group will be employees which use social media to exchange information online as part of the day to day jobs and duties in the organizations. An expectation of minimum 250 participants will revert on the online questionnaire which posted on the IBM Malaysia internal forum, since the forum currently has more than 1000 of active registered members. A $10 \%$ of the participants will be used to pilot test the instrument before actual survey takes place. The last step will be the data analysis using Structural Equation Modelling (SEM) as an analysis tool to analyse data collected, as SEM is a communicationsharing multivariate regression model that studies the influence of a factor to another factor.

Figure 1: Methodology Flowchart

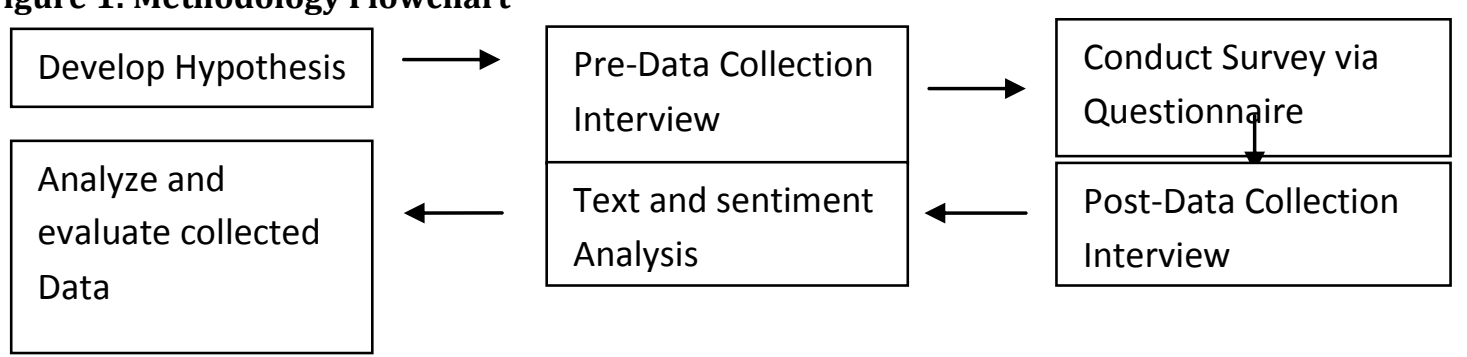

Research Hypotheses: The main objective of this research is to investigate whether the element of sentiment (Troit \& Pietermaritzburg, 2012; Tan et al., 2011) in a ego social network (Frantz \& Carley, 2009 ) is able to influence the collaboration and communication (Salome, 2009) attitude of knowledge workers when they share knowledge (Peariasamy \& Mansor, 2008) in the virtual Community of Practice (CoP) using Social Network Sites (SNS) in Knowledge-driven Companies. This study tests four hypotheses that perceived the relation between social media and sharing of knowledge:

H(1): The usage of social networking sites has positive relationship to knowledge sharing activity among knowledge workers within an organization.

Social networking sites enable knowledge workers to interact with others anytime from anywhere as long as Internet connection is available. More to the point, knowledge workers may utilize social network sites for knowledge sharing to solve their problematic working tasks even it is not within working hours.

H(2): The sentiment of social interaction ties between knowledge workers within an organization are positively associated with knowledge sharing via social network sites.

Social interaction tie is the connection that binds knowledge workers within an organization, and it exists whenever they exchange items or services. Social network sites keep workers connected all the time and serve as an important medium for them to share knowledge.

H(3): The sentiment of knowledge workers within an organization are positively associated with the quantity of knowledge sharing via social network sites.

The more frequent knowledge workers communicate or interact with each other on social network sites, the greater amount of knowledge will be shared. On the contrary, weak relationship due to less frequent dealing between each party may affect the amount of knowledge shared too.

H(4): The trust between knowledge workers within an organization is positively associated with their communication attitudes via social network sites.

Trust within interpersonal and inter-group relations strongly influences the quality and quantity of communication within an organization. The openness in accepting opinions from others serves as one of the factors that facilitate problems solving, and it is determined by the level of trust in oneself. Knowledge 
workers are more willing to pay attention and take in other's words when trust subsists, especially when an individual communicates in a nice or proper way with another individual. Good attitude forms trust and do have more power to influence others.

\section{Conclusion}

There are quite a number of research studies which shows that individuals have a preference to get constructive information and advice from other persons even though advance technologies are able to facilitate in knowledge investigation. Therefore, the right trust builders are necessitated especially in knowledge sharing among knowledge workers within an organization since trust is the main concern that supports knowledge-sharing in a workplace. Many organizations have started to work on various ways to smoothen the knowledge sharing procedure, for example using the social network analysis and also user level sentiment analysis to improve knowledge sharing via social network sites.

\section{References}

Abrams, L. C., Cross, R., Lesser, E. \& Levin, D. Z. (2003). Nurturing interpersonal trust in knowledgesharing networks. Academy of Management Executive, 17(4), 64-77.

Arnaboldi, V., Conti, M., Passarella, A. \& Pezzoni, F. (2012). Analysis of Ego Network Structure in Online Social Networks. 2012 International Conference on Privacy, Security, Risk and Trust and 2012 International Conference on Social Computing, 31-40. doi:10.1109/SocialCom-PASSAT.2012.41

Broughton, A., Higgins, T., Hicks, B. \& Cox, A. (2009). Workplaces and Social Networking - The Implications for Employment Relations.

Chua, R. Y., Ingram, P. \& Morris, M. W. (2005). Cognition- and Affect-based Trust in Managers' Professional Networks. Whom in Our Network Do We Trust?

Clark, A. V. (2005). Psychology of moods. (pp. 181 - 191). New York, NY: Nova Science Publishers Inc.

Connor, M. O. (2012). Professional vs. social networking. Retrieved from http://www.usatodayeducate.com/staging/index.php/career/professional-vs-socialnetworking

De Choudhury, M. (2011). Tie Formation on Twitter: Homophily and Structure of Egocentric Networks. Privacy, security, risk and trust (passat), 2011 IEEE third international conference on and 2011 IEEE third international conference on social computing (socialcom) , 465,470, 9-11 Oct. 2011

De Choudhury, M. \& Counts, S. (2013). Understanding affect in the workplace via social media. Proceedings of the 2013 conference on Computer supported cooperative work - CSCW 13, 303 316.

Fisher, D. (2005). Using Egocentric Networks to Understand Communication, 20-28.

Frantz, T. L. \& Carley, K. M. (2009). Using the CEMAP Facebook Profile in ORA. Harvesting Ego-Network Data from Facebook.

Gagne, M. (2009). A model of knowledge-sharing motivation, 48(4), 571-589. Doi: 10.1002/hrm

Ghalandari, K., Ghorbani, M., Jogh, G., Imani, M. \& Nia, L. B. (2012). The Effect of Emotional Labor Strategies on Employees Job Performance and Organizational Commitment in Hospital Sector. Moderating Role of Emotional Intelligence in Iran, 17(3), 319-326.

Hargadon, S. (2012). Educational Networking: The Important Role Web 2.0 Will Play in Education, pp. 1-5.

Hooff, B. V. D. \& Hendrix, L. (2004). Eagerness and Willingness to Share: The Relevance of Different Attitudes towards Knowledge Sharing.

Huy, Q. \& Shipilov, A. (2012). The key to social media success within organizations. MIT Sloan Review, 54(1), 73-81.

IBM Institute for Knowledge-Based Organizations. (2002). Using social network analysis to improve knowledge creation and sharing. A bird's-eye view.

Nandkeolyar, A. K. (2008). How do teams learn? Shared mental models and transactive memory systems as determinants of team learning and effectiveness.

Otte, E. \& Rousseau, R. (2002). Social network analysis: a powerful strategy, also for the information sciences. Journal of Information Science, 28(6), 441-453.

Peariasamy, T. \& Mansor, N. N. A. (2008). On-the-job Knowledge Sharing. How to Train Employees to Share Job Knowledge.

Rouse, M. (2012). Text Mining (Text Analytics). Retrieved from http://searchbusinessanalytics.techtarget.com/definition/text-mining 
Salome, M. R. (2009). Interpersonal Communication in the Workplace: Manager-Subordinate Relationships. Retrieved from http://voices.yahoo.com/interpersonal-communicationworkplace-manager-4865638.html

Sun, S., Ju, T. L., Chumg, H., Wu, C. \& Chao, P. (2009). Influence on Willingness of Virtual Community's Knowledge Sharing : Based on Social Capital Theory and Habitual Domain, 136-143.

Tan, C., Lee, L., Tang, J., Jiang, L., Zhou, M. \& Li, P. (2011). User-level sentiment analysis incorporating social networks. Proceedings of the 17th ACM SIGKDD international conference on Knowledge discovery and data mining - KDD '11, 1397. doi:10.1145/2020408.2020614

Wasko, M. M. \& Faraj, S. (2005). Why Should I Share? Examining Social Capital and Knowledge Contribution in Electronic Networks of Practice, MIS Quarterly, 29(1), 35.

Yuan, Y. C. \& Gay, G. (2006). Homophily of network ties and bonding and bridging social capital in computer-mediated distributed teams. Journal of Computer-Mediated Communication, 11(4), article 9 . 